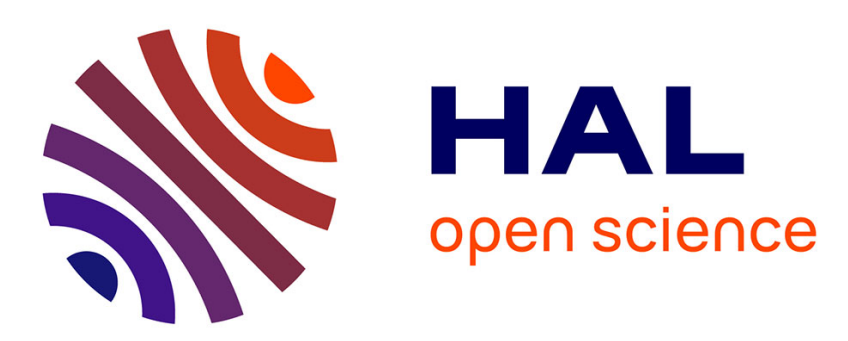

\title{
La représentation de la science est-elle genrée ? Étude auprès des étudiantes et étudiants inscrits en première année de filière scientifique à l'université de Lille
} Kristoff Talin, Anne-Frédérique Paul

\section{- To cite this version:}

Kristoff Talin, Anne-Frédérique Paul. La représentation de la science est-elle genrée ? Étude auprès des étudiantes et étudiants inscrits en première année de filière scientifique à l'université de Lille. Canadian Journal of Science, Mathematics and Technology Education, 2021, 21 (2), pp.456-474. 10.1007/s42330-021-00156-4 . hal-03470945

\section{HAL Id: hal-03470945 \\ https://hal.science/hal-03470945}

Submitted on 10 Dec 2021

HAL is a multi-disciplinary open access archive for the deposit and dissemination of scientific research documents, whether they are published or not. The documents may come from teaching and research institutions in France or abroad, or from public or private research centers.
L'archive ouverte pluridisciplinaire HAL, est destinée au dépôt et à la diffusion de documents scientifiques de niveau recherche, publiés ou non, émanant des établissements d'enseignement et de recherche français ou étrangers, des laboratoires publics ou privés. 


\section{LA REPRESENTATION DE LA SCIENCE EST-ELLE GENREE?}

\section{Étude auprès des étudiantes et étudiants inscrits en première année de filière scientifique à l’université de Lille}

Kristoff Talin

Chargé de recherche Centre National de la Recherche Scientifique (Clersé, Lille, France)

Anne-Frédérique Paul Antoire Maître de Conférences Université de Lille (France)

\section{Résumé :}

Les recherches sur les femmes dans la sphère scientifique sont nombreuses. Elles montrent, entre autres, que les hommes et les femmes ne s’inscrivent généralement pas dans les mêmes filières scientifiques. Au-delà de ce constat, il est pertinent de s’intéresser aux représentations des sciences de ces étudiantes et étudiants afin de déterminer ce qui influence leur choix. Cette recherche s’appuie sur une enquête menée auprès d’un échantillon représentatif de 1352 inscrits en première année de sciences à l'université de Lille. Nos résultats, tant quantitatifs que qualitatifs, soulignent l’importance des filières dans l'explication des différents modes de représentations des sciences, d'une part, et montrent que cette corrélation n'est pas due à la structure par genre des filières, d'autre part. Effectivement, que l'on soit du genre féminin ou masculin, on constate d'importantes disparités d'attitudes par rapport aux sciences, diversités qui s’avèrent fortement corrélées à la filière choisie. Il est donc important de relativiser l’influence du genre sur les modes de représentations des sciences.

Mots Clés : Genre, science, représentation, université, filière, France

Conflit d'intérêts: Les auteurs déclarent ne pas avoir de conflit d'intérêts. 


\section{INTRODUCTION}

Les recherches sur la présence des femmes dans la sphère scientifique sont nombreuses. Elles insistent souvent sur la faible présence du genre féminin dans un domaine porteur d’avenir (Buscatto et Marry 2009). Notre recherche se situe en aval de cette réflexion. Nous examinons en effet les représentations de la science parmi les étudiantes et étudiants inscrits à l’université en filière scientifique.

En 2016-2017 en France, les étudiants inscrits en licence scientifique représentaient 25,1 \% des étudiants inscrits à l’université, soit environ 250000 étudiants (Note d’Information 2017). Les filles forment 40,1 \% du total et sont donc sous-représentées, alors qu’elles sont surreprésentées en santé, en arts, en lettre et langues ainsi que dans les sciences humaines et sociales (SHS). Ces études scientifiques sur les inscriptions genrées en filière scientifique sont pertinentes, mais une recherche complémentaire s'intéressant aux représentations ${ }^{1}$ des sciences selon le genre nous paraît tout aussi nécessaire. Elle permettra de savoir si, à niveau de présence égal dans le cursus universitaire, le genre a une incidence sur ces représentations. La différence ou la similitude des représentations est, en effet, importante pour expliquer la moindre présence des femmes dans la sphère scientifique.

La recherche sur les représentations des sciences présentée dans cet article est le fruit d’un travail d'analyse d’une enquête par questionnaire. Ce sondage a été mené auprès d'un échantillon représentatif de 1352 inscrits en première année de sciences à l’université de Lille, tant dans la filière générale (licence) que technologique (IUT) ${ }^{2}$. 700 sont inscrits dans les thématiques concernant les sciences physiques et de l’ingénieur, alors que 590 suivent une formation axée sur les sciences biologiques. Le questionnaire a été passé in situ lors de la réunion de rentrée. Les étudiants avaient le choix de répondre ou non à ce questionnaire, ce qui respecte les normes éthiques définies en France par la Commission nationale de l'informatique et des libertés (CNIL).

\footnotetext{
${ }^{1}$ Le terme représentation est utilisé ici dans le sens que lui confèrent Jodelet et Moscovici. Pour Jodelet, les représentations sont des «modalités de pensée pratique orientées vers la communication, la compréhension et la maîtrise de l'environnement social, matériel et idéal » (Jodelet, 2003, p. 371). La fonction d'une représentation est de " rendre quelque chose d'inhabituel ou l'inconnu lui-même, familier » (Moscovici, 1976, p. 38).

${ }^{2}$ Sur les 2175 inscrits, 1900 ont été contactés et 1352 ont répondu (ainsi, le taux de réponse est de 62 \%). Par manque de place dans cet article, la présentation détaillée des enquêtés et le questionnaire employé - tout comme le type d’analyses qualitative et quantitative menées - ne sont pas présentés dans le chapitre.
} 
Cette recherche a aussi comme particularité le fait que le questionnaire comportait à la fois des questions « fermées » et des questions « ouvertes » sur la définition de la science, le niveau d’intérêt que les participants et participantes entretenaient pour les sciences et leurs représentations des sciences $^{3}$. Ainsi, les analyses présentées s'appuieront sur la comparaison des résultats quantitatifs et qualitatifs. Après un état de la littérature sur ces questions, nous présenterons, dans la seconde partie de l’article, les résultats de la recherche.

\section{LE GENRE FEMININ ET LES SCIENCES : UN COUPLE EN TENSION}

Alors qu'elles réussissent mieux que les hommes dans les études scientifiques, les femmes sont pourtant moins nombreuses à exercer dans le domaine (Bulletin d’information de l’ISU 2015).

\subsection{La place des femmes dans les professions scientifiques}

D’une manière générale, les travaux de sociologie montrent que les différences de genre perdurent de façon durable pour l'accès et la carrière dans les professions supérieures (Buscatto et Marry 2009) ${ }^{4}$. En 2015, à peu près dans tous les pays présentés par l’Institut de statistique de l’UNESCO (ISU), la part des femmes scientifiques est inférieure à 50 \% (Bulletin d'information de l’ISU 2015). En Europe occidentale, ce pourcentage est de $32 \%$ et la France fait figure de mauvais élève avec 25,6 \% ${ }^{5}$. Selon Smuck (2014), les résultats sont similaires pour les ingénieurs et le reste des professions scientifiques de niveau au moins égal à 14 années d'études complétées. Cette auteure souligne que les femmes dans les métiers scientifiques insistent sur leurs difficultés à concilier vie professionnelle et vie de famille. Des aménagements du travail (télétravail, temps flexibles ou partiels, mais aussi garderies) pourraient ainsi être des leviers puissants pour féminiser ces professions.

\footnotetext{
${ }^{3}$ La définition et le niveau d’intérêt nous informent aussi sur les représentations des sciences.

${ }^{4}$ Le terme « professions supérieures » reprend l’idée développée et employée par l’Institut national de la statistique et des études économiques quand elle qualifie les «professions intellectuelles et les cadres supérieurs d'entreprises et de la fonction publique », dont les professions scientifiques, les médecins...

${ }^{5}$ Seul le Luxembourg affiche un pourcentage de femmes chercheuses inférieur à celui de la France.
} 
De la même manière, les femmes quittent davantage ces secteurs d'activité avec l'âge, sans pour autant que des facteurs explicatifs ne soient avancés. Par ailleurs, on note une forte « dis-parité » ${ }^{6}$ de genre concernant la satisfaction au travail (Enquête Gender Scan 2017).

Certes, la situation professionnelle des femmes évolue, mais des «dis-parités » perdurent. Des facteurs professionnels et liés aux responsabilités domestiques peuvent les expliquer. Mais ces éléments objectifs ne sont pas les seuls explicatifs. Ainsi, la représentation que se font les personnes des sciences peut influer à tout moment de la carrière, mais aussi en amont, lors du choix du programme d'études.

Par ailleurs, les femmes sont encore, à poste égal, moins bien rémunérées que les hommes, ont des progressions de carrière moins intéressantes et des niveaux de responsabilité inférieurs que les leurs. En prenant l'exemple des biologistes, des sociologues cherchent à expliquer les raisons de cette différence (Marry et Jonas 2005). Elles mettent en évidence le rapport à la maternité comme facteur explicatif en insistant sur sa dimension subjective.

Dans aucun pays sans doute, on ne trouve aujourd’hui aucun lien de causalité directe entre la réussite professionnelle des femmes chercheuses ou universitaires et la présence d'enfants, mais, sur le plan subjectif, le conflit travail/famille est partout très fort. Sans doute, parce que les chercheuses partagent à la fois l’éthos wébérien de la dévotion totale à « la » science et l’idéal de mère, dévouée et disponible à son (ses) enfant(s). (Ibid. p.

Prendre en compte cet ethos wébérien dans l'analyse, c’est nécessairement considérer le concept de représentation dans l'analyse des rapports entre genre et sciences. C’est dans cette filiation théorique que nous ancrons notre recherche. En effet, ces représentations de la science peuvent avoir un impact sur les choix de carrière et les stratégies professionnelles. De la même manière, elles peuvent avoir un effet sur les choix disciplinaires (par exemple, le secteur de la biologie est plus féminisé que le

\footnotetext{
${ }^{6}$ En écrivant « dis-parité » sous cette forme, nous souhaitons insister sur le fait que la différence entre les femmes et les hommes dans le domaine scientifique se fait toujours au détriment de la place des femmes, donc s'éloigne d'une vision paritaire des faits.
} 
secteur de l'informatique) ou sur les professions scientifiques exercées (les femmes sont particulièrement sous-représentées dans la recherche scientifique).

Par exemple des représentations des sciences, plus axées sur la définition et l'appréhension du vivant que sur la dimension technique et les innovations technologiques, prédisposeront à des choix de carrières valorisant davantage la connaissance du vivant et donc à orienter vers des métiers proches des sciences biologiques. Plus largement, les représentations des sciences influent en amont sur le choix de la filière de formation. Ces deux facteurs influant, de manière concomitante, sur le choix d'une carrière.

\subsection{Les représentations des sciences : deux genres différents?}

Les recherches concernant les représentations des sciences existent, mais elles sont moins développées que celles concernant la place des femmes dans les sciences, d'une part, et elles portent peu sur l'articulation entre genre et représentation des sciences, d'autre part. L'étude de Boy et Chanvril porte sur les technologies du vivant. Elle montre que les femmes sont plus prudentes dans l'utilisation de ces technologies et apparaissent plus critiques par rapport à l'usage qui en est fait. Il y a donc un effet du genre sur la représentation des technologies du vivant (Boy et Chanvril 2016). L’article de Mariotti étudie les représentations sociales de la science d’élèves du secondaire, âgés de 11 à 18 ans (Mariotti 2003). D’après les conclusions de ces études, des effets de genre sont observables et des représentations sexuées de la science s’observent dès le secondaire (notamment au niveau de l’intérêt pour la science qui diffère entre les filles et les garçons, ces derniers se montrant plus intéressés par cette discipline) . Dès la première année du secondaire, les filles se représentent la science en insistant sur le vivant (avec des thèmes relatifs à la biologie ou la médecine) alors que pour les garçons, l’organisation de la représentation se fait autour de la physique et de l'objet technique (Ibid. p. 604). Dans la même coulée théorique et empirique, Deschênes montre en 2002 que ces affirmations trouvent aussi leur validation en terre québécoise.

Un mémoire de maîtrise montre que les étudiantes se destinant à l'enseignement au primaire sont insuffisamment formées dans le domaine des sciences et de la culture technique (Lisée 2008). Des 
entretiens montrent que leur manière d'enseigner cette culture est largement corrélée à leurs représentations des sciences. Ceci permet de suggérer que les représentations des enseignantes peuvent renforcer les biais genrés des représentations des sciences chez les élèves ${ }^{7}$. D’autres facteurs peuvent renforcer ces biais genrés (par exemple les illustrations et les rôles attribués dans les manuels scolaires). Ainsi, les représentations des sciences chez les élèves et les étudiants sont des constructions complexes, fruit d'une histoire personnelle, de représentations sociales et scolaires.

\subsection{Hypothèses pour rendre compte de la « dis-parité » du genre en science}

Les recherches menées sur le couple « genre et science » montrent des résultats tout en contrastes. Alors que les filles réussissent mieux dans les filières scientifiques à partir de la classe de seconde ${ }^{8}$, elles sont pourtant moins nombreuses à intégrer des écoles d’ingénieurs, mais aussi des filières scientifiques de l’Université (Blanchard et al. 2014). Les femmes sont aussi proportionnellement moins nombreuses dans les professions scientifiques, aussi bien dans le secteur privé que public. Celles qui œuvrent en recherche scientifique publient moins d'articles que leurs homologues masculins et accèdent proportionnellement moins à des postes de prestige ou de décision (Larivière et al. 2013). Les politiques publiques et des groupes d'intérêt font pourtant la promotion et le développement d’actions pour favoriser la présence des femmes en sciences en commençant dès le primaire, avec le choix des illustrations dans les manuels (Un plan pour les sciences et technologies 2011). Ces actions ne donnent pas, pour l’instant en France, les résultats escomptés. Ce constat semble d’ailleurs généralisé, quel que soit le pays occidental concerné. Les recherches au sujet des représentations des sciences peuvent offrir une approche explicative complémentaire pour comprendre le phénomène de « dis-parité » de genre en sciences.

C’est dans cette foulée sociologique que s’ancre l'objet central de cet article dans lequel nous développons deux hypothèses centrales :

- $\quad$ Hypothèse 1 : l'existence de niveaux d'intérêts différents pour la science entre les hommes et les femmes ;

\footnotetext{
${ }^{7}$ Les personnes qui enseignent au primaire sont, de façon majoritaire, des femmes.

${ }^{8}$ Soit le secondaire 4 au Québec.
} 
- Hypothèse 2 : ces différences de niveaux d’intérêt s’accompagnent de définitions et de représentations différentes des sciences.

Les travaux mentionnés ci-dessus, auxquels se rattachent ces deux hypothèses, sont riches d’enseignement sur l’importance des représentations, mais ils ont toutefois une portée globalisante. En effet, ils ne prennent pas en compte la discipline scientifique choisie (plus près des sciences physiques et de l’ingénieur ou encore plus près des sciences biologiques) pour expliquer les différentes représentations des sciences. C’est pourquoi nous faisons une hypothèse complémentaire.

- Hypothèse 3 : Dès le collège, les disciplines scientifiques ainsi que les filières ne véhiculent pas la même représentation des sciences et ces différences sont susceptibles de modifier les représentations des sciences parmi les étudiantes et les étudiants.

L’articulation de ces différentes hypothèses pourrait s’agencer de la façon suivante (Figure 1).

$\underline{\text { Figure } 1: \text { Genre filière et rétroaction des représentations }} \underline{9}$

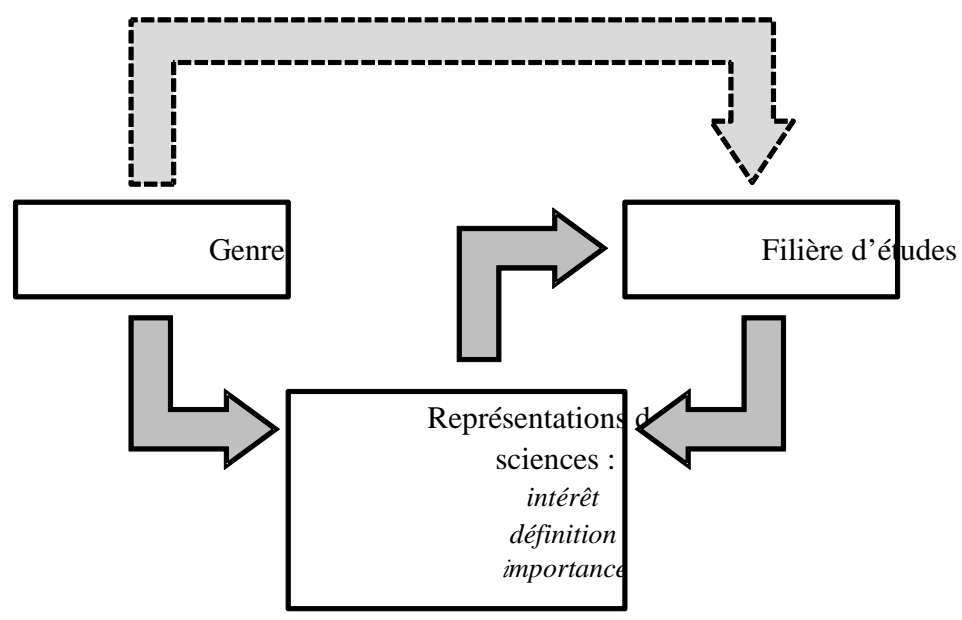

\section{GENRE, FILIERE ET REPRESENTATIONS IDEALISEES DES SCIENCES ?}

\subsection{Méthodologie de l'enquête par questionnaire}

Le questionnaire a été élaboré à partir de trois sources principales :

- des questions issues des Eurobaromètres sur le thème de la science et des technologies ;

\footnotetext{
${ }^{9}$ La flèche en pointillé présente un effet, mais un effet moindre que les flèches en traits pleins. Mesurer l'intérêt et l'importance de la science ainsi qu'observer la définition de la science permet d'appréhender les représentations dans plusieurs dimensions. Ces trois dimensions sont fréquentes dans l'étude des représentations en sociologie (voir par exemple Michelat et Simon, 1977) et permettent d'avoir une approche assez complète des représentations de l'objet d'études.
} 
- $\quad$ des questions provenant d'enquêtes sur les ingénieurs ${ }^{10}$;

- des questions élaborées par nos soins concernant la biologie, la biologie de synthèse, la chimie et la chimie verte et issues d’un travail préparatoire d’entretien avec les étudiants en L1 de ces licences.

Il a ensuite été testé auprès de 20 étudiants, ce qui a facilité la réalisation d'un questionnaire comprenant 61 questions articulées autour de sept thèmes :

1. questions concernant les études et les projets professionnels ;

2. opinions sur la science en général ;

3. opinions à propos de la biologie et de la biologie de synthèse ;

4. opinions concernant la chimie et la chimie verte ;

5. opinions sur des problèmes de société ;

6. questions au sujet de l'avenir ;

7. questions sociodémographiques.

L’article porte sur des questions concernant les deux premiers thèmes du questionnaire.

Les réponses aux questions fermées 5, 8, 9, $10^{11}$ ont fait l’objet d'analyses univariées et bivariées. Des tests de significativité, ainsi que des calculs de coefficients de corrélation ont systématiquement été réalisés.

Les réponses à la question ouverte 6 Pour vous, qu'est-ce que la science ? ont été saisies manuellement et ont fait l'objet d'une analyse statistique associant les fréquences des mots et expressions les plus couramment utilisés (en regroupant les mots de racine identique) et une analyse de concordance. Cette analyse a permis d'amorcer la construction d'une grille de thèmes pour faire une analyse de contenu.

\footnotetext{
${ }^{10}$ Enquête annuelle des Ingénieurs et scientifiques de France (ISF) parue en 2011 (https://www.iesf.fr/)

${ }^{11}$ Voir la méthodologie détaillée dans l'annexe méthodologique disponible en ligne.
} 
La population étudiée permet d'examiner les représentations des sciences auprès d’un public encore relativement novice en sciences. Nous sommes face à un groupe à l'intersection de trois temps de la vie :

- $\quad$ il amorce la phase de transition entre le statut d’étudiant et celui de professionnel ;

- $\quad$ il est en transition entre des représentations initiales de la science (fruit de leur histoire, leur environnement et des représentations des sciences promues par l'école) et leurs transformations par l’instance de socialisation et d'enseignement que représente l’université ;

- $\quad$ il est en phase de transition entre l’image de la science véhiculée par l'école et celle véhiculée par l’université, sachant que l’école vise à enseigner « la science pour tous » alors que l'université vise à enseigner «la science pour quelques-uns ». En effet, l'école vise l'ensemble de la population des élèves, avec le lycée général, alors que l'université vise une fraction des étudiants et est divisée en filières spécialisées (secteurs définis par les arrêtés licence et master), mais avec des cursus propres à chaque université (diplômes propres à chaque université).

La répartition des genres dans les différentes filières n’est pas équivalente. Ainsi, dans notre échantillon, la filière relatives aux sciences physique et de l'ingénieur comporte 83 \% d’hommes et 17 \% de femmes, alors qu'en filière des sciences biologiques, il y a 62 \% de femmes contre 38 \% d’hommes $^{12}$. La filière des sciences biologiques est donc plus diversifiée que celle des sciences physiques et de l’ingénieur et ces deux filières présentent un rapport entre sexes inversé.

\subsection{Un intérêt pour les sciences qui varie selon le genre et les filières}

Le niveau d'intérêt pour la science est mesuré sur une échelle de 1 à 10 et la définition de la science fait l’objet d'une question ouverte à laquelle 71 \% des enquêtés ont répondu.

\footnotetext{
${ }^{12}$ Cette répartition est représentative de l'ensemble des inscrits à l’Université de Lille en filière sciences physiques et de l’ingénieur ou en sciences biologiques.
} 
La moyenne d’intérêt - 7,60 - est élevée. Seulement 7 \% des répondants donnent une note inférieure ou égale à 5. Il n'en demeure pas moins que la distribution des réponses est relativement normale (graphique 1).

\section{Graphique 1 : Niveau d'intérêt pour les sciences (en pourcentage)}

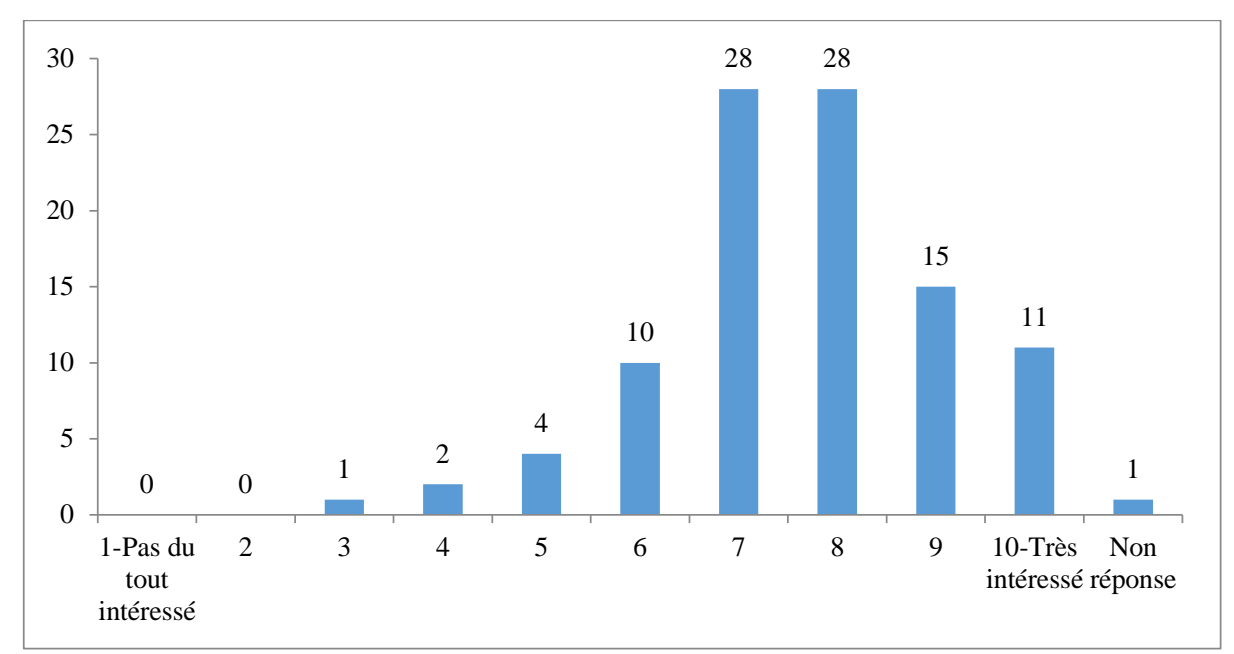

La moyenne des réponses varie selon la filière des répondants alors que le sexe n’est pas statistiquement significatif. Les inscrits en sciences physiques et de l’ingénieur ont une moyenne moins élevée $(7,52)$ que ceux en sciences biologiques $(7,75)^{13}$. Qu'en est-il de l'effet cumulé du genre et de la filière ? Le tableau 1 montre que l'effet cumulatif genre et filière existe, mais qu'il joue de manière moins évidente qu’attendu. En effet, l’intérêt le plus fort est déclaré dans la filière des sciences biologiques, mais davantage chez les hommes. C'est l'inverse en sciences physiques et de l’ingénieur, où ce sont les femmes qui ont la plus forte moyenne.

Tableau 1: Moyenne d'intérêt pour la science selon le genre et la filière (Analyse de variance significative au seuil de 0,01 )

\begin{tabular}{|l|c|}
\hline Moyenne & 7,60 \\
\hline Homme sciences biologiques & 7,85 \\
Femme sciences biologiques & 7,68 \\
Femme sciences physique et de & 7,53 \\
l'ingénieur & \\
\hline
\end{tabular}

\footnotetext{
${ }^{13}$ Analyse de variance significative au seuil de 0,006.
} 


\begin{tabular}{|l|l|} 
Homme sciences physiques et de & 7,46 \\
l'ingénieur & \\
\hline
\end{tabular}

Le groupe ayant le plus haut niveau d'intérêt est le genre minoritaire dans chacune de ces deux filières. Le choix d'une filière considérée comme allant moins de soi (sciences biologiques pour les hommes, sciences physiques et de l’ingénieur pour les femmes) est donc corrélé avec un intérêt pour la science plus important. Le choix de cette filière «à contre-genre » peut donc apparaître davantage comme revendiqué que comme consensuel, et peut provenir d’un intérêt accru pour la science.

Par ailleurs, une forte corrélation s'observe entre le niveau d'intérêt pour la science et l'intérêt pour les débats scientifiques. L’intérêt scientifique s’accompagne donc d'un intérêt pour les activités associées aux sciences ${ }^{14}$. Les questions 8,9 et 10 sont fortement corrélées deux à deux, si bien qu'il est possible de construire un indice cumulatif permettant de classer les individus en profils en fonction de leur niveau d’intérêt pour le domaine scientifique. Les résultats du tableau 2 témoignent d’un fort niveau d'intérêt, puisque plus d'un enquêté sur deux obtient le score d'intérêt maximum (56 \%) et que seulement $7 \%$ ont le score minimum.

$\underline{\text { Tableau } 2 \text { : Niveau d'intérêt, en pourcentage, pour le domaine scientifique selon le genre et la filière }}{ }^{15}$

\begin{tabular}{|l|c|c|c|c|}
\hline & Intérêt -- & Intérêt - & Intérêt + & Intérêt ++ \\
\hline Moyenne & 7 & 14 & 23 & 56 \\
\hline Homme sciences biologiques & 4 & 13 & 20 & 64 \\
Femme sciences biologiques & 4 & 16 & 23 & 58 \\
Homme sciences physiques et de l'ingénieur & 8 & 14 & 25 & 53 \\
Femme sciences physiques et de l'ingénieur & 13 & 15 & 21 & 52 \\
\hline
\end{tabular}

${ }^{14}$ Q8. Êtes-vous intéressé par les sujets ou les débats scientifiques? Q9. Vous informez-vous sur les sujets ou les débats scientifiques?

Q10. D’une manière générale, par rapport aux découvertes scientifiques, êtes-vous enthousiaste, pas enthousiaste, indifférent ? La construction de l'indice est expliquée dans l'annexe méthodologique.

${ }^{15} \mathrm{Chi}^{2}$ significatif au seuil de 0,01 . V de Cramer de 0,14. 
Ces pourcentages ne sont pas très surprenants, dans la mesure où cette population est inscrite dans des filières scientifiques. Les inscrits en filière « biologique » sont plus intéressés que ceux suivant la filière " physique et ingénierie ». Par rapport aux résultats de cet indice, la filière suivie est plus explicative du niveau d'intérêt que le genre, ce qui confirme les résultats précédents. Il ne faut toutefois pas nier l'incidence du genre sur le niveau et les formes d'intérêts pour le domaine scientifique, mais c’est la filière choisie qui est la plus explicative.

\subsection{Une définition polysémique des sciences}

Une question ouverte permettait aux interviewés de définir ce qu’est la science dans leurs propres mots $^{16}$. $71 \%$ des enquêtés ont répondu, ce qui témoigne d'un niveau d’intérêt élevé pour cette question. Les étudiants répondent significativement plus que les étudiantes (78\% versus $63 \%$ ) tout comme les inscrits en sciences physiques et de l'ingénieur par rapport à la filière des sciences biologiques (78 \% versus 64 \%). Cette « dis-parité » peut relever du même type de biais que celui observé à l'école : les filles s’abstenant dans le doute (Mosconi, 1998). Comme pour les questions précédentes, le pourcentage de réponses varie fortement selon le genre et la filière (tableau 3).

Tableau 3 : Répartition, en pourcentage, des enquêtés à la question 6 selon le genre et la filière ${ }^{17}$

\begin{tabular}{|l|c|c|}
\hline & Réponse à la question & Non-réponse à la question \\
\hline Ensemble de l'échantillon & 71 & 29 \\
\hline Homme sciences physiques et de l'ingénieur & 82 & 18 \\
Femme sciences physiques et de l'ingénieur & 74 & 26 \\
Homme sciences biologiques & 71 & 29 \\
Femme sciences biologiques & 60 & 40 \\
\hline
\end{tabular}

Les différences cumulatives entre la filière, d'une part, et le genre, d'autre part, sont assez nettes et les analyses renforcent l'importance de la filière comme critère le plus explicatif. Quel que soit le genre, les inscrits en sciences physiques et de l’ingénieur répondent le plus à la question, les inscrites

\footnotetext{
${ }^{16}$ Q6. Pour vous, qu'est-ce que la science?

${ }^{17} \mathrm{Chi}^{2}$ significatif au seuil de 0,000. V de Cramer de 0,21.
} 
en sciences biologiques le moins, ce qui contribue à valider les hypothèses de recherche 1 et 3 . Le genre a, certes, une importance, mais celui-ci apparaît nettement moins explicatif que la filière. En effet, les étudiants inscrits en sciences physiques et de l’ingénieur - qu’ils soient hommes ou femmes - répondent plus à la question que ceux inscrits en sciences biologiques.

On peut donc en déduire que le genre est moins explicatif que la filière. Autrement écrit, qu’il soit femme ou homme, c'est d'abord l'inscription en filière des sciences physiques et de l'ingénieur qui prédispose à la réponse libre concernant la définition des sciences. Ce résultat pourrait s'expliquer par la nature des disciplines enseignées. Les sciences physiques et de l’ingénieur se rapprochent plus de l'idéal des sciences - voire incarnent le paradigme des sciences - tout à la fois exactes et expérimentales. En revanche, en sciences biologiques, les disciplines étudiées, bien que relevant du domaine des sciences «dures », sont plus éloignées du modèle idéalisé des sciences (par la complexité de leurs objets, leur moindre mathématisation des modèles, leur plus faible pouvoir prédictif ou leurs conditions d’observation ou d'expérimentation). Six dimensions principales ressortent de l'analyse.

\section{La définition des sciences : entre objectivité, complexité et exaltation}

À travers la fréquence des mots choisis par les étudiants et relevant des mêmes champs lexicaux, trois axes principaux de définition peuvent être distingués :

1. la compréhension (du monde) est la thématique la plus fréquente (34 \% des répondants mentionnent les mots comprendre ou compréhension dans leur réponse) ;

2. la capacité à agir sur le monde apparaît aussi dans un quart des réponses (24 \% des répondants mentionnent les verbes pouvoir ou permettre) ;

3. l'étude des phénomènes naturels est également évoquée (les mots étude ou étudier apparaissent dans $23 \%$ des réponses) ${ }^{18}$ et pour étudier le monde, si l’observation et l’expérimentation sont très peu spontanément mentionnées, la recherche et la découverte le sont plus fréquemment, mais ont un statut ambigu entre le moyen d'étude et moyen

\footnotetext{
${ }^{18}$ Cette analyse de fréquence a été confortée par une relecture du contexte de ces termes pour s’assurer qu’il n’y a pas de contresens (négations...).
} 
d'intervention. Ces faibles valeurs sont à mettre en relation avec le fait que la question portait sur « qu'est-ce que la science » et pas sur « comment fait-on de la science ». Pourtant, on observe que dans nombre de réponses, les étudiants définissent la science par la manière d'en faire.

Les mots dérivés de «étude/étudier » sont plutôt associés à «phénomènes » alors que ceux dérivés de compréhension/comprendre sont plutôt corrélés avec «monde ». On peut citer pour exemple que la science, c'est « l'étude des phénomènes naturels » ou encore qu' « elle amène à la compréhension de ce que nous sommes et de ce qui nous entoure du progrès ».

Certains étudiants expriment aussi le fait que la science permet de comprendre la dynamique du monde (17 \%) et l'évolution du vivant (19\%) alors que d'autres précisent que cette compréhension se fait, quel que soit l’objet étudié. Certains étudiants ont donc une vision dynamique du monde (qui est alors vu comme un système, multidimensionnel et animé de processus dynamiques) ${ }^{19}$ alors que d'autres ont une vision plus figée (décrire le monde, comme par un instantané versus un film) ou plus déterministe du monde (et donc aisément mathématisable, modélisable ${ }^{20}$ sous forme de lois ou de théories même si le dualisme théorique / pratique est évoqué) ${ }^{21}$.

Une minorité d'étudiants expriment une vision relativiste ou tout au moins évolutive des sciences, ce qui correspond à la vision épistémologique actuelle des sciences (Kuhn, 1972), mais aussi à sa présentation scolaire dans les programmes. Ainsi, le préambule des programmes de physique-chimie des classes de seconde générale et premières scientifique, littéraire ou économique (élèves de 17 ou 18 ans) indique que «Contrairement à la pensée dogmatique, la science n’est pas faite de vérités révélées intangibles, mais de questionnements, de recherches et de réponses qui évoluent et

\footnotetext{
${ }^{19}$ Exemples : «C'est un ensemble de disciplines (astronomie, mécanique...) qui permettent de comprendre le fonctionnement de l'univers, de comprendre l'infiniment petit et l'infiniment grand »,

« Une manière de comprendre les lois de la nature, l'évolution du monde et le faire évoluer », «Étude de tout ce qui nous entoure de sa composition de son évolution ».

${ }^{20}$ Exemples : « C’est pour moi un moyen de prouver des propos théoriques et de conduire à l'avancement d'une civilisation »,

« Découverte du monde qui nous entoure par la justification de théories »,

« La science est l'étude de la vie et des lois physiques qui jouent sur la matière remarquable grâce à certains phénomènes ou expériences à l'aide d'une vision rationnelle et mathématique ».

${ }^{21}$ Exemples : « Science est un ensemble de connaissances théoriques et pratiques dans un domaine spécifique »,

« La recherche, la théorie, la pratique ».
} 
s'enrichissent avec le temps » (Bulletin officiel, 2010). Le socle commun de connaissances, de compétences et de culture ${ }^{22}$, précise quant à lui que «La démarche scientifique a pour objectif d'expliquer l'Univers, d'en comprendre les évolutions, selon une approche rationnelle distinguant les faits et les hypothèses vérifiables d'une part, les opinions et les croyances d’autre part. ».

On peut citer quelques exemples de réponses des étudiants :

- « Il ne faut pas en avoir une idée préconçue : la science est une notion évolutive qui ne cesse d'innover les formes qui pourraient la définir. Ce sont la découverte, la recherche, l’analyse et la révolution »;

- « Pour moi, la science est un secteur pédagogique vaste qui attend encore d'être exploré en profondeur même si nous pensons connaître beaucoup de choses dans le secteur scientifique. Je suis certain que nous ne connaissons qu'une infime partie de ce qui nous entoure » ;

- «Un domaine en constante expansion qui regroupe plusieurs spécialités visant à simplifier le quotidien de l'être humain ».

D’autres réponses témoignent de la difficulté à définir la science «C’est quelque chose, apparemment... » ou bien encore une définition qui met l'accent sur son importance et sur sa large présence, sans toutefois la décrire davantage : « une science est la matière la plus importante dans le monde nous la retrouvons partout ». Pour ces étudiants, le concept de science n’est donc pas identifié, mais il est reconnu comme ayant une importance.

La définition des sciences n'est pas seulement un exercice d’objectivation. La question « Pour vous, qu'est-ce que la science ? » génère aussi des déclarations assez exaltées. Ainsi, certains expriment un rapport subjectif à la science, qu'il s’agisse d'une dimension affective : «C'est une étoile qui nous donne de la lumière quand on est dans le sombre » ou proche de la croyance positiviste : «Un domaine permettant de découvrir des vérités sur le monde, les hommes, etc., et cherchant à aider le

\footnotetext{
${ }^{22}$ D’après le Ministère de l’Éducation, de la Jeunesse et des Sports, le socle commun de connaissances, de compétences
} et de culture présente ce que tout élève doit savoir et maîtriser à 16 ans. 
progrès » ou d’inspiration philosophique : « Une discipline qui se rapproche le plus de la vérité ». Les termes vérité et vrai suggèrent que pour certains étudiants, « la science dit le vrai », ce en quoi nombre de scientifiques et d’épistémologues s’inscrivent en faux (Barreau, 2016).

\section{La science spontanée associée aux technologies, au progrès et aux professions scientifiques}

Certains étudiants associent spontanément science et technologie : « La science va de pair avec les nouvelles technologies et est donc un vecteur d'innovateurs ». Parfois, l'association est si forte qu'on atteint la substitution : «Une technique complexe qui permet d'orienter l'homme dans sa vie, de le guider, de l'aider et d'améliorer ses conditions de vie. La science est également une marque du passé, elle représente le présent, mais aussi le futur ».

Pour ces étudiants, la dimension « agir sur le monde » prend le pas sur la dimension « comprendre le monde ». On peut interpréter ce type de représentation comme un glissement des sciences vers la technoscience conçue comme un «ensemble dans lequel coopèrent institutions, chercheurs et ingénieurs afin de mettre en œuvre, pour des applications précises, les ressources de la science et de la technique $»^{23}$.

Dans la logique de l'assimilation entre la science et ses applications technologiques, la dimension du progrès pour soi et pour l'humanité est très présente dans les discours ${ }^{24}$, même quand on leur demande de définir la science. Science et progrès sont très fortement associés dans un discours qui n’est pas loin du positivisme scientifique qui a dominé le $19^{\mathrm{e}}$ siècle et une partie du $20^{\mathrm{e}}$ siècle (Gingras, 2018). Des étudiants associent la science à des métiers «scientifiques », mais qui ne relèvent pas sensu stricto d'une activité de production des sciences. Ils sont davantage ancrés dans une activité d’application qui implique le recours à des savoirs, des méthodes, des principes, des appareils issus des sciences. « Progrès médical, recherche pour l'amélioration du niveau de vie ».

\footnotetext{
${ }^{23}$ https://www.larousse.fr/dictionnaires/francais/technoscience/76967

${ }^{24}$ Exemples : « La science est synonyme de progrès ! Elle permet d'expliquer notre monde, de révolutionner dans les domaines médicaux. », « La découverte de nouvelles choses, un enseignement qui sert au progrès »,

« C’est un phénomène qui permet d'expliquer des faits concrets et abstraits, la science permet d'avoir des connaissances pour développer le futur ».
} 
On peut donc noter ici une certaine confusion entre l'activité de recherche (chercheurs, certains ingénieurs) et celle d’application de productions scientifiques (techniciens, médecins) qui ne sont pas institutionnellement des scientifiques, mais qui a une activité souvent considérée comme celle de scientifiques.

\subsection{Vers des définitions genrées?}

La définition des sciences, sa séparation en disciplines et sa contribution à la compréhension du monde varient selon le genre.

Définir la science et comprendre le monde

La perception du monde et des sciences varie avec le genre. Ainsi, les femmes privilégient les êtres vivants et la vie alors que les hommes mentionnent davantage l'environnement. Formulé autrement, c’est une opposition entre le système, d'une part, et ses composantes, d'autre part. Nous sommes donc face à deux représentations significativement différentes des sciences.

Si la science permet de comprendre le monde, ce que cela veut dire diffère avec le genre. Pour les femmes, comprendre permet d'expliquer, de dire comment le monde fonctionne, comment il a émergé et changé alors que pour les hommes, comprendre permet surtout de répondre à des questions. En revanche, pour toutes et tous, la science permet la formulation de lois. Le modèle de la physique, comme idéal de la scientificité, est donc très fort.

Les hommes semblent avoir des représentations plus « utilitaires », voire utilitaristes, des sciences que les femmes, ce qui peut suggérer une vision plus positiviste des premiers. Les femmes semblent avoir des représentations plus cognitives des sciences, mais elles évoquent aussi le pourquoi, ce qui est explicitement une question à laquelle ne peut répondre la science... Ainsi, les idées - pourtant combattues par les scientifiques eux-mêmes - d'une science source de vérité permettant de « dire le pourquoi » restent présentes dans cette population qui se destine à des professions scientifiques. 


\section{La science disciplinarisée}

La science n’est pas identifiée aux mêmes disciplines. En effet, si la physique et la chimie sont des références partagées, les hommes mentionnent plus les mathématiques et les femmes plus la biologie. La perception de la diversité des disciplines est plus marquée chez les femmes.

Les hommes sont, quant à eux, proportionnellement sous-représentés dans les disciplines qu’ils ne mentionnent pas, tout comme les femmes d'ailleurs. Les femmes sont peu présentes en physique, mais très présentes en biologie et mentionnent peu la physique et plus la biologie. Plusieurs explications peuvent être apportées :

- $\quad$ leur sous-représentation dans les parcours mathématiques (sauf pour spécifiquement devenir enseignant) ;

- $\quad$ le poids de l’idée reçue - constituant sans aucun doute une représentation sociale très prégnante - qui postule que les filles, selon une idée largement répandue dans la société française, ne sont « pas faites pour les maths »;

- $\quad$ leur surreprésentation en sciences biologiques.

Une étude comparative - entre le genre et la filière - des mots évoqués montre que les proximités entre les femmes et les hommes sont fortes au sein d'une même filière. Ainsi, même si les étudiants de la filière des sciences physiques et de l’ingénieur mettent en avant les disciplines et apparaissent plus positivistes que les étudiantes, ils partagent l’idée selon laquelle la science doit comprendre le monde qui nous entoure. En sciences biologiques, si les étudiantes insistent davantage sur les êtres humains et les étudiants sur l'environnement et le vivant, ils soulignent ensemble que la science doit permettre de comprendre la vie et la Terre.

Ces éléments d'analyse permettent de conclure que les inscrits au sein d'une même filière, indépendamment de leur genre, ont des réponses plus homogènes que les hommes ou les femmes de filière différentes. Ce n’est pas pour autant que le genre n’a pas d’incidence, mais il est moins explicatif que la filière. Ainsi, plus que le genre, la filière d'inscription semble contribuer à forger des différences de définitions et de représentations des sciences. 


\section{CONCLUSION}

Notre recherche reposait sur des hypothèses axées sur la «dis-parité » des représentations des sciences selon le genre : d'une part, il y a des niveaux d’intérêts différents pour la science entre les hommes et les femmes; d'autre part, les différences de niveaux d'intérêt s'accompagnent de définitions et de représentations différentes des sciences. Ces hypothèses liées au genre ont été complétées par la suivante : les disciplines scientifiques contribuent, dès l'école, à véhiculer des représentations différentes susceptibles de modifier les représentations initiales.

Nous constatons que les étudiants et les étudiantes conçoivent les sciences différemment, tant au niveau de la définition des sciences qu'au niveau de l’importance qui leur est conférée. Cette première conclusion nous permet de valider la seconde hypothèse.

Nous n’avons pas de données suffisamment précises sur la position socio-économique des étudiants eux-mêmes, pour tester une hypothèse qui proposerait une articulation entre des représentations différenciées des sciences et le positionnement social ${ }^{25}$.

Comme nous avons constaté une sexualisation des filières, il n’est pas possible de négliger l'hypothèse selon laquelle le genre est un paramètre qui contribue au choix de la filière. Cependant, aux vues de nos résultats, il nous paraît plus important d'insister sur la complémentarité de la filière et du genre sur les représentations des sciences.

L'apport principal de notre recherche est la validation de l'hypothèse soulignant l'importance des filières suivies, par les étudiantes et les étudiants, pour expliquer les différents modes de représentations des sciences. À bien des égards, la filière choisie est corrélée avec les représentations des sciences. Et, comme nous l'avons vérifié dans toutes les analyses, cette corrélation n’est pas due à la structure par genre des filières. Autrement dit, ce n'est pas parce que les femmes sont fortement surreprésentées en sciences biologiques et que les hommes le sont en sciences physiques et de l’ingénieur que l’on peut expliquer les différences de représentations observées selon les filières.

\footnotetext{
${ }^{25}$ Notamment dans le cadre des polémiques techno-scientifiques qui postulent souvent un déficit de connaissance.
} 
Effectivement, que l'on soit du genre féminin ou masculin, on constate d’importantes « dis-parités » d’attitudes par rapport aux sciences, variations qui s’avèrent fortement corrélées à la filière suivie.

Cette recherche contribue à renforcer l’idée selon laquelle les approches quantitative et qualitative ne sont pas opposées, mais complémentaires. Nous montrons, en effet, que les résultats de l'analyse de questions fermées - traitées de manière quantitative - convergent avec ceux de l’analyse de la question ouverte « Pour vous, qu'est-ce que la science ? ». Il y a toujours un impact du genre et un impact de la filière dans la formulation des réponses. Cette convergence des résultats, provenant de deux méthodes d'enquête différentes, renforce leur pertinence et la force des conclusions que nous pouvons en tirer.

Au moment de conclure cette recherche, il convient de rappeler que nous avons montré que parmi les étudiants universitaires de première année en science à l’Université de Lille, les perceptions des sciences sont diverses et que cette diversification est fortement associée au genre. Nous avons aussi montré que la filière d'études a un effet sur ces perceptions des sciences, et pourrait conforter les différences initiales de représentations liées au genre.

Nous n’affirmons pas, pour autant, que la filière d’études surdétermine le genre dans la construction des représentations des sciences, mais nous pouvons, au moins, affirmer qu’il est nécessaire de prendre en compte - indépendamment du genre - la filière d'études pour comprendre la manière dont se composent et se structurent les représentations des sciences. Au vu du caractère relativement novateur de ce thème dans la recherche en France, nous manquons de résultats qui permettraient d'affiner ces conclusions. Deux thèmes au moins seraient à traiter pour accroître la validité de ces résultats :

- développer des recherches empiriques sur la même thématique dans d'autres universités, ce qui permettrait de lever le biais lié à un effet local ;

- $\quad$ s’intéresser aux motivations des acteurs pour le choix de leur filière afin de mieux connaître les raisons de l’importance de cette variable dans l’explication des représentations des sciences. 


\section{Bibliographie}

Barreau, A. (2016). De la vérité dans les sciences. Paris: Dunod.

Beaudry, C. et al. (2016). Which gender gap? Factors affecting researchers' scientific impact in science and medicine. Research Policy, 45(9), 1790-1817.

Blanchard, M. et al. (2014). La production d'une noblesse scientifique: Enquête sur les biais de recrutement à l'ENS. Paris: École Normale Supérieure.

Boy, D. et Chanvril, F. (2010). Les représentations sociales des technologies du vivant en Europe. Sociologie et sociétés, 42(2), 17-42.

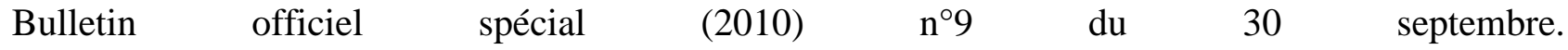
https://www.education.gouv.fr/pid24426/special-n-9-du-30-septembre-2010.html. Consultation: 13 Juillet 2020.

Buscatto, M. et Marry, C. (2009). Le plafond de verre dans tous ses éclats. La féminisation des professions supérieures au XX $X^{\mathrm{e}}$ siècle. Sociologie du travail, 51(2), 170-182.

Deschênes, C. (2002). Les femmes, la science, l’ingénierie et la technologie. Recherches féministes, 15(1), 1-6.

Enquête Gender scan. (2017). Global Conseil. http://www.global-contact.net/wordpress/wpcontent/uploads/2017/10/Pr\%C3\%A9sentation_Gender-Scan-2017.pdf. Consultation: 10 Novembre 2018.

Gingras, Y. (2018). Histoire des sciences. Paris: Presses universitaires de France.

Jodelet, D. (2003). Les représentations sociales. Paris: Presses universitaires de France.

Keller, E. F. (2003). Gender and Science. In S. Harding et M. Hintikka (Eds.), Discovering Reality. Feminist Perspectives on Epistemology, Metaphysics, Methodology, and Philosophy of Science (pp. 187-205). Dordrecht: Springer.

Kuhn, T. (1972). La structure des révolutions scientifiques. Paris: Flammarion. 
Larivière, V. et al. (2013). Global gender disparities in science. Nature, 504(7479), 211-213.

Le socle commun de connaissances, de compétences et de culture (2018), Ministère de l’Education nationale, de la Jeunesse et des Sports. https://www.education.gouv.fr/le-socle-commun-deconnaissances-de-competences-et-de-culture-12512.Consultation: le 10 Juillet 2020.

Leleux, J. P. et Olivier, M. (2014). Faire connaître et partager les cultures scientifique, technique et industrielle : un impératif, Office Parlementaire d’évaluation des choix scientifiques et technologiques. https://www.senat.fr/rap/r13-274/r13-2741.pdf. Consultation: 25 Novembre 2018. Les femmes en sciences. (2015). Bulletin d'information de l'ISU, N³4.

Lisée, V. (2008). Représentations sociales de l'importance des sciences et technologies et de la culture scientifique et technologique dans l'enseignement et la formation à l'enseignement primaire au Québec chez des futures enseignantes. Sherbrooke: Mémoire présenté à la Faculté d'éducation.

Mariotti, F. (2002). La sexuation de l’implication à s’engager dans un métier scientifique au lycée. Recherches féministes, 15(1), 47-63.

Mariotti, F. (2003). Structures comparées des représentations sociales de la science et des métiers scientifiques chez les collégiens et les lycéens des deux sexes. In M. Lavallée (Ed.). Les constructions sociales. Constructions nouvelles. (pp. 589-607). Montréal: Geirso. http://www.geirso.uqam.ca/zancien_site/livre_repres_sociales/index_livre.php. Consultation: 7 Octobre 2018.

Marry, C. et Jonas, I. (2005). Chercheuses entre deux passions. L’exemple des biologistes. Travail, genres et sociétés, 14(2), 69-88.

Michelat, G. et Simon, M. (1977). Classe, religion et comportement politique. Paris: Presses de la Fondation nationale des sciences politiques.

Mosconi, N. (1998). Réussite scolaire des filles et des garçons et socialisation différentielle des sexes à l'école. Recherches féministes, 11(1), 7-17. 
Moscovici, S. (1976). La psychanalyse, son image et son public. Paris: Presses universitaires de France.

Note d'information. (2017). Ministère de l'Enseignement supérieur, de la Recherche et de l'Innovation http://cache.media.enseignementsup-recherche.gouv.fr/file/2018/47/2/NI_17.13__Etudiants_universites_2016-2017_875472.pdf. Consultation: 12 Octobre 2018.

Schmuck, C. (2014). Rapport Mutationnelles 14. Formations et emplois des femmes dans les sciences et technologies en France. http://www.global-contact.net/wordpress/wpcontent/uploads/2014/09/Mutationnelles-2014_VF.pdf. Consultation: 10 Octobre 2018.

Un plan pour les sciences et technologies. (2011). Ministère de l’Éducation nationale http://eduscol.education.fr/cid54734/un-plan-pour-les-sciences-et-technologies.html. Consultation: 20 Octobre 2018.

Une nouvelle ambition pour les sciences et les technologies à l'École. (2011). Bulletin officiel, 10, 10 mars http://www.education.gouv.fr/cid55255/mene1105413c.html. Consultation: 17 Octobre 2018. 


\section{ANNEXE METHODOLOGIQUE}

Cette annexe présente la population étudiée (1), le questionnaire (2) et les principes et sources suivies pour l'analyse des questions ouvertes (3).

Il convient, pour commencer, de préciser que l'ensemble de cette recherche s’est effectuée selon le protocole mis en place par la Commission Nationale de l'Informatique et des Libertés (CNIL). Cette commission contrôle, en autres, que la gestion d'une enquête auprès d'une population respecte le droit à la liberté des individus concernés par une enquête sociologique. Elle s’assure que le questionnaire ne contient pas de données sensibles et vérifie que les enquêtés soient libres de répondre.

\section{LA POPULATION ETUDIEE}

La majeure partie des inscrits en première année scientifique a pu être interrogée (certains n’ont pu l'être, car ils n'étaient pas présents le jour de passation de l'enquête). Ainsi, sur les 2175 inscrits, 1900 ont été contactés soit 87 \%. Le taux de réponse est en moyenne de 68 \%. Il est particulièrement élevé à l’IUT et dans la filière SVTE et il est toujours supérieur à 50 \%.

La comparaison de l'échantillon avec les données exhaustives de 2013 - fournies par l'Observatoire des formations et de l'insertion professionnelle (OFIP) de l'Université de Lille 1 - montre qu'il est représentatif de l'ensemble des inscrits en filière scientifique. Par exemple, la répartition des inscriptions selon le genre est proche : les hommes sont majoritaires dans l'ensemble (62\% contre $65 \%$ pour l’OFIP) avec une prédominance à l'IUT (71\% contre 75 \% pour l'OFIP). Sans être parfaitement en adéquation avec les données de l’OFIP, les résultats sur le genre de notre enquête sont représentatifs des inscrits en licence.

La même vérification a été faite pour la filière d'inscription des étudiants. Au sein des filières Sesi et Svte, la proportion de femmes dans l'échantillon est assez proche des données de l'OFIP. Ainsi, l'échantillon comporte $17 \%$ de femmes inscrites en Sesi (contre $20 \%$ dans les données de l'OFIP) et $61 \%$ inscrites en Svte (contre $57 \%$ dans les données de l’OFIP). La validité de l'échantillon se confirme, y compris au sein des deux filières étudiées.

En ce qui concerne l'âge des répondants, l'échantillon est sensiblement plus jeune (18,9 ans contre 19,1 ans). Cette surreprésentation des plus jeunes est particulièrement sensible chez les 18 ans et moins (52\% contre $43 \%$ ) et touche davantage les inscrits en licence que ceux inscrits à l'IUT. Pour ces derniers, en effet, les différences avec l'enquête OFIP 2013 sont non significatives. La moyenne d’âge moins élevée de l'échantillon en licence peut résulter du fait que notre questionnaire a été passé lors des réunions de prérentrée dans chacune des filières considérées. Lors de ces réunions, les redoublants - donc plus âgés - sont sans doute moins présents que les primo-entrants, ce qui contribue 
à faire baisser la moyenne d’âge des répondants. Un pourcentage conforte cette hypothèse : l’âge moyen des inscrits dans les filières IUT est similaire à celui de l’OFIP.

En conclusion, les répondants à notre enquête apparaissent, sur plus d'un point, similaires à ceux des enquêtes fournies dans l'enquête OFIP de 2013. Elles confirment par exemple qu'hommes et femmes ne s’inscrivent pas dans les mêmes filières. De même, les origines sociales des étudiants ne sont pas sans lien avec la filière dans laquelle ils s’inscrivent. Mais surtout, elles tendent à légitimer la qualité de représentativité de l'échantillon obtenu. Si la structure de la population, qui a répondu en 2014, est proche des données exhaustives de 2013 fournies par l’OFIP, cela signifie que l'échantillon que nous avons interrogé représente bien l'ensemble des inscrits en filière scientifique à Lille 1.

\section{LE QUESTIONNAIRE}

\subsection{Conception}

Le questionnaire a été élaboré à partir de trois sources principales :

- des questions issues des Eurobaromètres sur le thème de la science et des technologies ;

- $\quad$ des questions provenant d'enquêtes sur les ingénieurs ${ }^{26}$;

- $\quad$ des questions élaborées par nos soins concernant la biologie, la biologie de synthèse, la chimie et la chimie verte et issues d’un travail préparatoire d'entretiens avec les étudiants en L1 de ces licences.

Le questionnaire a ensuite été testé auprès de 20 étudiants, ce qui nous a permis de vérifier la compréhension des différents items proposés. Ce test a facilité la réalisation d'un questionnaire comprenant 61 questions articulées autour de sept thèmes :

- $\quad$ questions concernant les études et les projets professionnels ;

- $\quad$ opinions sur la science en général ;

- $\quad$ opinions à propos de la biologie et de la biologie de synthèse ;

- $\quad$ opinions concernant la chimie et la chimie verte ;

- $\quad$ opinions sur des problèmes de société ;

- $\quad$ questions au sujet de l'avenir ;

- $\quad$ questions sociodémographiques.

\footnotetext{
${ }^{26}$ Enquête annuelle des Ingénieurs et scientifiques de France (ISF) parue en 2011 (https://www.iesf.fr/)
} 
L’article porte sur des questions concernant les deux premiers thèmes du questionnaire.

Texte du questionnaire

Bonjour, ceci est une étude scientifique concernant vos opinions et vos attentes sur la science et ses applications. Elle est organisée par une équipe d'enseignants-chercheurs en sciences expérimentales et en sociologie de Lille 1.

Répondre à ce questionnaire, pas très long et anonyme, est une contribution utile à la compréhension des choix de filières et des orientations professionnelles dans les matières scientifiques et technologiques.

Les résultats seront d'autant plus représentatifs, intéressants et donc utiles que vous serez nombreux à y répondre. Ils pourront aussi être utilisés pour améliorer les enseignements et mieux répondre à vos attentes professionnelles.

Pour indiquer votre choix, il vous suffit d'entourer le chiffre correspondant à votre réponse

Voici, pour commencer, quelques questions concernant vos études et vos projets professionnels.

1. Dans quelle formation êtes-vous actuellement inscrit?

IUT Chimie 1

IUT Génie Biologique $\quad 2$

IUT Génie Electrique \& Informatique Industrielle 3

IUT Génie Mécanique \& Productique $\quad 4$

IUT Informatique 5

$\begin{array}{ll}\text { IUT Mesures Physiques } & 6\end{array}$

$\begin{array}{ll}\text { Chimie } & 7\end{array}$

Electronique, Energie électrique, Automatique $\quad 8$

Génie civil 9

$\begin{array}{ll}\text { Informatique } & 10\end{array}$

Mathématiques 11

Mathématiques, informatique appliquées aux sciences humaines et sociales 12

Mécanique 13

$\begin{array}{ll}\text { Physique } & 14\end{array}$

$\begin{array}{ll}\text { Physique-Chimie } & 15\end{array}$

$\begin{array}{ll}\text { Sciences de la Terre } & 16\end{array}$

$\begin{array}{ll}\text { Sciences de la vie } & 17\end{array}$

Sciences de la vie et de la Terre $\quad 18$ 


\section{L’année dernière, étiez-vous :}

En terminale

A Lille 1, dans la même filière

A Lille 1, dans une autre filière, si oui dans quelle formation

Ailleurs, si oui, dans quelle formation :

\section{Quelle sont les raisons principales qui ont conduit à vous inscrire dans cette formation ?}

Parce que j'aime la discipline

Parce que la discipline m'avait plu au lycée

Parce que cela correspondait vraiment à ce que je voulais faire

Parce que cette formation offrait un maximum de possibilités de réorientation

4

Parce que on me l'a fortement suggéré à l'école (enseignant, conseiller ...)

Parce que je ne savais pas vraiment où m'inscrire

Parce que j'ai été refusé dans une autre filière

Parce que j'ai échoué dans une autre filière

Parce que je ne voulais pas entrer directement dans la vie active

4. Aujourd'hui, diriez-vous que vous avez un projet professionnel défini ? (Donnez une note de 1 à 10, où 1 signifie que vous avez un projet pas du tout défini et 10 signifie que vous avez un projet très nettement défini)

Pas du tout défini

Tout à fait défini
J'ai un projet professionnel...
1
$\begin{array}{llllllll}2 & 3 & 4 & 5 & 6 & 7 & 8 & 9\end{array}$
10 
Nous aimerions, à présent, connaître vos opinions concernant la science en général.

5. Dans l'ensemble, quel est votre niveau d'intérêt pour la science en général ? (Donnez une note de 1 à 10 où 1 signifie que vous n'êtes pas du tout intéressé et 10 signifie que vous êtes très intéressé) Pas du tout intéressé Très intéressé
Je suis...
1
23
45
$\begin{array}{llll}6 & 7 & 8 & 9\end{array}$
10

\section{Pour vous, qu'est-ce que la science?}

7. Et pour chacun des domaines suivants, les sciences sont-elles importantes?

$\begin{array}{lcccc} & \text { Oui } & \text { Non } & \text { Je ne sais pas } \\ \text { pour mes études pour ma carrière } & 1 & 2 & 3 \\ \text { pour la vie de tous les jours pour la santé } & 1 & 2 & 3 \\ \text { pour l'environnement pour le progrès } & 1 & 2 & 3 \\ \text { pour comprendre le monde } & 1 & 2 & 3\end{array}$

8. Etes-vous intéressé par les sujets ou les débats scientifiques?

Très intéressé

Assez intéressé

Peu intéressé

Pas du tout intéressé 
9. Vous informez-vous sur les sujets ou les débats scientifiques?

Oui, très souvent

Oui, la plupart du temps $\quad 2$

Oui, de temps en temps 3

Non, rarement 4

Non, jamais 5

10. D’une manière générale, par rapport aux découvertes scientifiques, êtes-vous :

Plutôt enthousiaste $\quad 1$

Plutôt pas enthousiaste $\quad 2$

Indifférent 3

11. Et par rapport aux nouvelles applications technologiques, êtes-vous :

Plutôt enthousiaste $\quad 1$

Plutôt pas enthousiaste $\quad 2$

Indifférent 3

12. En dehors de la formation que vous suivez actuellement :

Souvent Parfois Rarement Jamais

Suivez-vous l’information scientifique dans les médias ? $\quad 1 \quad 3 \quad 3 \quad 4$

Suivez-vous sur des sites internet à vocation $1-2$

scientifique?

Suivez-vous sur des sites généralistes pour y chercher de

l'information autour des sciences ?

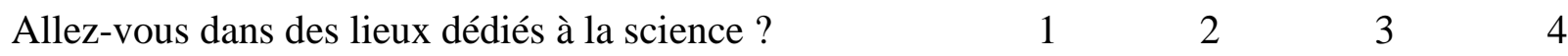

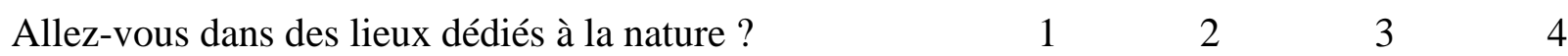

Allez-vous dans des clubs ou des associations en lien avec

les sciences?

$\begin{array}{llll}1 & 2 & 3 & 4\end{array}$

Pratiquez-vous avec quelqu'un de votre famille ou un(e) ami(e) une activité en lien avec les sciences ?

$\begin{array}{lll}2 & 3 & 4\end{array}$




\section{Après vos études, aimeriez-vous exercer une profession liée aux sciences et aux technologies}

?

Oui 1

Non

Je ne sais pas

Voici à présent quelques propositions qui concernent la biologie.

\section{La biologie vous intéresse-t-elle?}

Beaucoup

Assez

Un peu

Pas du tout

15. Selon vous, la biologie aide-t-elle à comprendre les enjeux, les problèmes et les possibilités en matière... (Donnez une note de 1 à 10, où 1 signifie qu'elle n'aide pas et 10 signifie qu'elle aide beaucoup)

\begin{tabular}{|c|c|c|c|c|c|c|c|c|c|c|}
\hline & 1 - Elle n’aide pas & 2 & 3 & 5 & 5 & 6 & 7 & 8 & 9 & 10 - Elle aide beaucoup \\
\hline d'alimentation & 1 & 2 & 3 & 4 & 5 & 6 & 7 & 8 & 9 & 10 \\
\hline de santé & 1 & 2 & 3 & 4 & 5 & 6 & 7 & 8 & 9 & 10 \\
\hline de reproduction & 1 & 2 & 3 & 4 & 5 & 6 & 7 & 8 & 9 & 10 \\
\hline d’énergie & 1 & 2 & 3 & 4 & 5 & 6 & 7 & 8 & 9 & 10 \\
\hline de climat & 1 & 2 & 3 & 4 & 5 & 6 & 7 & 8 & 9 & 10 \\
\hline de biodiversité & 1 & 2 & 3 & 4 & 5 & 6 & 7 & 8 & 9 & 10 \\
\hline de pollution & 1 & 2 & 3 & 4 & 5 & 6 & 7 & 8 & 9 & 10 \\
\hline d'environnement & 1 & 2 & 3 & 4 & 5 & 6 & 7 & 8 & 9 & 10 \\
\hline
\end{tabular}


16. Selon vous, la biologie aide-t-elle à comprendre comment fonctionne le corps humain ?

Oui tout à fait 1

Oui plutôt 2

Non plutôt pas 3

Non pas du tout 4

Je ne sais pas 5

17. Vous arrive-t-il de suivre les informations concernant la biologie ?

$\begin{array}{cccc}\text { Souvent } & \text { De temps en temps } & \text { Rarement } & \text { Jamais } \\ 1 & 2 & 3 & 4 \\ 1 & 2 & 3 & 4 \\ 1 & 2 & 3 & 4 \\ 1 & 2 & 3 & 4\end{array}$

18. En règle générale, la biologie est-elle utile pour faire des choix en matière...

Oui tout à fait Oui plutôt Non plutôt pas Non pas du tout Je ne sais

Dans les émissions de télévision

Dans les journaux

Sur internet

Dans des musées, des clubs...

pas

d'alimentation

1

2

3

4

5

de santé

1

2

3

4

5

d'environnement

2

3

5

d'énergie

2

3

5

de sexualité

1

2

3

5 
19. Selon vous, ces applications issues de la biologie sont-elles plutôt porteuses de craintes ou d'espoirs?

la vaccination

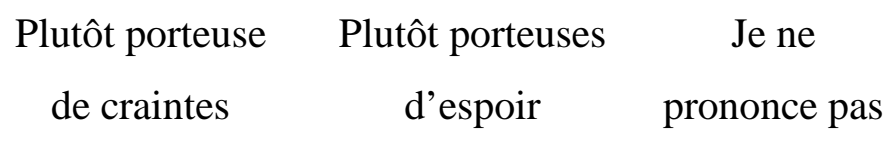

les antibiotiques

1

2

3

la thérapie génique

1

2

3

la thérapie cellulaire (cellules souches,

organes de remplacements)

les organismes génétiquement modifiés

1

2

3

la procréation médicalement assistée

1

2

3

les techniques liées à la fin de vie

1

2

3

$\begin{array}{lll}1 & 2 & 3\end{array}$

le séquençage d'ADN

1

2

3

l’imagerie cérébrale

1

2

1

2

3

20. Pour vous, qu'est-ce que la biologie ?

21. Avez-vous déjà entendu parler de la biologie de synthèse ?

Oui

1 (Si vous répondez oui, continuez le questionnaire)

Non

2 (Si vous répondez non allez directement à la question 28) 


\section{Où en avez-vous entendu parler?}

dans vos cours à l’Université

Oui Non

dans vos cours au lycée

12

dans les journaux, à la radio ou à la télévision

12

au cinéma

12

sur internet

12

dans des conférences, des débats...

12

dans une association

12

12

23. Selon-vous qu'est-ce que la biologie de synthèse ?

24. Pensez-vous que la biologie de synthèse soit utile pour l'avenir?

Oui tout à fait

Oui plutôt 2

Non plutôt pas 3

Non pas du tout 4

Je ne sais pas

25. Pensez-vous que la biologie de synthèse puisse présenter des risques pour l'avenir?

Oui tout à fait

Oui plutôt 2

Non plutôt pas 3

Non pas du tout

Je ne sais pas

26. Selon vous, serait-il bon que la biologie de synthèse soit utilisée pour :

améliorer l'homme

Oui Non

Je ne sais pas

produire de nouveaux OGM

12

interfacer le vivant et l'informatique

12

12


produire de nouveaux biocarburants

1

résoudre la pollution

12

3

fabriquer des produits nouveaux

1

2

3

27. Etes-vous d'accord ou non avec les affirmations suivantes :

La biologie de synthèse est prometteuse

La biologie de synthèse promet beaucoup mais permet peu

La biologie de synthèse joue avec le feu

Oui Non Je ne sais pas

$\begin{array}{lll}1 & 2 & 3 \\ 1 & 2 & 3 \\ 1 & 2 & 3\end{array}$

28. Que pensez-vous que l’appellation « biologie de synthèse " puisse désigner ?

Voici à présent quelques opinions concernant la chimie.

\section{La chimie vous intéresse-t-elle ?}

Beaucoup

Assez

Un peu

Pas du tout

30. Selon vous, la chimie aide-t-elle à comprendre les enjeux, les problèmes et les possibilités en matière... (Donnez une note de 1 à 10, où 1 signifie qu'elle n'aide pas et 10 signifie qu'elle aide beaucoup)

$\begin{array}{lcccccccccc} & 1 \text { - Elle n'aide pas } & 2 & 3 & 5 & 5 & 6 & 7 & 8 & 9 & 10 \text { - Elle aide beaucoup } \\ \text { d'alimentation } & 1 & 2 & 3 & 4 & 5 & 6 & 7 & 8 & 9 & 10 \\ \text { de santé } & 1 & 2 & 3 & 4 & 5 & 6 & 7 & 8 & 9 & 10 \\ \text { d'énergie } & 1 & 2 & 3 & 4 & 5 & 6 & 7 & 8 & 9 & 10 \\ \text { de matériaux } & 1 & 2 & 3 & 4 & 5 & 6 & 7 & 8 & 9 & 10 \\ \text { d'environnement } & 1 & 2 & 3 & 4 & 5 & 6 & 7 & 8 & 9 & 10\end{array}$


31. Selon vous, la chimie aide-t-elle à comprendre comment fonctionne le corps humain ?

Oui tout à fait 1

Oui plutôt

Non plutôt pas 3

Non pas du tout

Je ne sais pas

32. Vous arrive-t-il de suivre les informations concernant la chimie ?

Souvent De temps en temps Rarement Jamais

Dans les émissions de télévision

1

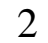

3

4

Dans les journaux

1

2

3

4

Sur internet

1

2

3

4

Dans des musées, des clubs...

1

2

3

4

33. En règle générale, la chimie est-elle utile pour faire des choix en matière...

Oui tout à fait Oui plutôt Non plutôt pas Non pas du tout Je ne sais

$\begin{array}{llllll} & & & & \text { pas } \\ \text { d'alimentation } & 1 & 2 & 3 & 4 & 5 \\ \text { de santé } & 1 & 2 & 3 & 4 & 5 \\ \text { d'environnement } & 1 & 2 & 3 & 4 & 5 \\ \text { d'énergie } & 1 & 2 & 3 & 4 & 5 \\ \text { de matériaux } & 1 & 2 & 3 & 5\end{array}$

34. Selon vous, ces applications issues de la chimie sont-elles plutôt porteuses de craintes ou d'espoirs?

Plutôt porteuse de Plutôt porteuses Je ne prononce craintes d'espoir pas

l’hygiène et la santé

1

2

3

les médicaments

1

3

la détection de toxiques ou de polluants

1

3

la conservation des aliments

1

3

l'habillement

1

3

la production de carburants

1

la réduction des toxiques et des 1

polluants 
36. Avez-vous déjà entendu parler de la chimie verte ?

Oui

1 (Si vous répondez oui, continuez le questionnaire)

Non

2 (Si vous répondez non allez directement à la question 44)

\section{Où en avez-vous entendu parler ?}

dans vos cours à l’Université

Oui Non

dans vos cours au lycée

12

dans les journaux, à la radio ou à la télévision

12

au cinéma

12

sur internet

12

dans des conférences, des débats...

12

dans une association

$\begin{array}{ll}1 & 2 \\ 1 & 2\end{array}$

38. Selon-vous qu'est-ce que la chimie verte ?

39. Pensez-vous que la chimie verte soit utile pour l'avenir?

Oui tout à fait

Oui plutôt

Non plutôt pas

Non pas du tout

Je ne sais pas 
40. Pensez-vous que la chimie verte puisse présenter des risques pour l’avenir?

Oui tout à fait

Oui plutôt

Non plutôt pas

Non pas du tout

Je ne sais pas

41. Voici une série d'affirmations que l'on entend à propos de la chimie verte. Etes-vous ou non d'accord avec elles ?

\begin{tabular}{|c|c|c|c|}
\hline & Oui & Non & $\begin{array}{c}\text { Je ne sais } \\
\text { pas }\end{array}$ \\
\hline C'est une chimie moins toxique pour l'homme? & 1 & 2 & 3 \\
\hline C'est une chimie moins polluante ? & 1 & 2 & 3 \\
\hline $\begin{array}{l}\text { C'est une chimie qui n'est pas moins polluante mais qui fait de } \\
\text { la pub en prétendant être moins polluante? }\end{array}$ & 1 & 2 & 3 \\
\hline $\begin{array}{l}\text { C'est une chimie qui transforme des substances d'origine } \\
\text { biologiques? }\end{array}$ & 1 & 2 & 3 \\
\hline C’est une chimie basée sur des ressources renouvelables? & 1 & 2 & 3 \\
\hline C’est une chimie qui produit des produits nouveaux ? & 1 & 2 & 3 \\
\hline C’est une chimie pour le développement durable ? & 1 & 2 & 3 \\
\hline C'est une chimie qui promet beaucoup mais n'aboutit à rien ? & 1 & 2 & 3 \\
\hline
\end{tabular}

42. Que pensez-vous que l'appellation « chimie verte " puisse désigner ? 
Afin de mieux analyser vos réponses, nous aimerions connaitre votre opinion sur des problèmes de société.

43. Quel impact ont les sciences et les technologies sur :

$\begin{array}{lcccc} & \text { Un impact plutôt } & \text { Un impact plutôt } & \text { Pas } & \text { Je ne sais } \\ & \text { positif } & \text { négatif } & \text { d'impact } & \text { pas } \\ \text { la santé } & 1 & 2 & 3 & 4 \\ \text { l'alimentation } & 1 & 2 & 3 & 4 \\ \text { le climat } & 1 & 2 & 3 & 4 \\ \text { l'environnement } & 1 & 2 & 3 & 4 \\ \text { l'agriculture } & 1 & 2 & 3 & 4 \\ \text { l'industrie } & 1 & 2 & 3 & 4 \\ \text { les loisirs et la culture } & 1 & 2 & 3 & 4 \\ \text { le logement } & 1 & 2 & 3 & 4 \\ \text { les transports } & 1 & 2 & 3 & 4 \\ \text { l'habillement } & 1 & 2 & 3 & 4 \\ \text { les objets de tous les jours } & 1 & 2 & 3 & 4 \\ \text { les emballages } & 1 & 2 & 3 & 4\end{array}$


44. Concernant le traitement des déchets nucléaires, quel est l'avis le plus proche du vôtre ? C'est un problème face auquel il suffit de trouver une solution technique 1

Il faut trouver une solution technique mais il faut aussi qu'elle soit débattue 2 publiquement

Ce n'est pas vraiment un problème mais comme les gens sont inquiets, il faut les informer

Ce n'est pas vraiment un problème et les gens sont suffisamment informés

45. Concernant les organismes génétiquement modifiés, quel est l'avis le plus proche du vôtre ? C'est un problème face auquel il suffit de trouver une solution technique 1 Il faut trouver une solution technique mais il faut aussi qu'elle soit débattue 2 publiquement

Ce n'est pas vraiment un problème mais comme les gens sont inquiets, il faut les informer

Ce n'est pas vraiment un problème et les gens sont suffisamment informés

46. De manière générale, le progrès technique apporte à l'Homme :

Plus de mal que de bien 1

Plus de bien que de mal 2

A peu près autant de mal que de bien 3

Je ne sais pas 4 
47. Pour l'avenir, il est important...

$\begin{array}{lcccc} & \begin{array}{c}\text { Très } \\ \text { important }\end{array} & \begin{array}{c}\text { Assez } \\ \text { important }\end{array} & \begin{array}{c}\text { Peu } \\ \text { important }\end{array} & \begin{array}{c}\text { Pas important } \\ \text { du tout }\end{array} \\ \begin{array}{l}\text { de réduire notre empreinte écologique } \\ \text { de favoriser le développement }\end{array} & 1 & 2 & 3 & 4 \\ \text { d'innovations technologiques } & 1 & 2 & 3 & 4 \\ \text { de favoriser les approches de type } & 1 & 2 & 3 & 4 \\ \text { "développement durable" } & & & 4 \\ \text { de mieux surveiller les impacts des } & 1 & 2 & 3 & 4 \\ \begin{array}{l}\text { sciences et technologies } \\ \text { de redévelopper des techniques } \\ \text { traditionnelles }\end{array} & 1 & 2 & 3 & 4 \\ \text { d’informer les gens sur les avancées } & 1 & 2 & 3\end{array}$

48. Dans la vie quotidienne, avez-vous spontanément le réflexe...

$\begin{array}{lcc} & \text { Oui } & \text { Non } \\ \text { d'éteindre les lumières inutiles } & 1 & 2 \\ \text { d'utiliser du papier recyclé } & 1 & 2 \\ \text { d'acheter des produits "verts" } & 1 & 2 \\ \text { d'éviter la voiture pour de courtes distances } & 1 & 2 \\ \text { de trier vos ordures ménagères } & 1 & 2 \\ \text { de réduire le chauffage lorsqu'il fait trop chaud } & 1 & \end{array}$

Voici trois questions concernant votre avenir...

\section{L’année prochaine souhaitez-vous :}

Poursuivre dans la même formation que celle que vous suivez aujourd'hui ?

Aller dans une autre formation scientifique ou technique ? 2

Changer d'orientation? 3

Je ne sais pas $\quad 4$ 
50. Réussir pour vous, c'est d'abord... (Vous pouvez choisir le nombre de réponses que vous désirez.)

Gagner beaucoup d'argent

Agir sur le plan culturel, social ou politique

Avoir des infants

Crier one entreprise 4

Exercer un métier intéressant

Innover dans le domaine technique

Etre indépendant

Trouver un équilibre affectif

Prendre part aux avancées scientifiques

Faire carrière

51. Voici la liste de quelques traits qui peuvent être considérés comme importants pour une activité professionnelle. Quels sont ceux qui vous paraissent les plus importants ? (Vous pouvez choisir le nombre de réponses que vous désirez.)

On gagne bien sa vie

On a des horaires satisfaisants

On ne risque pas le chômage

On peut espérer une promotion

On a un métier qui est bien considéré

On a de l'initiative

On a un métier qui est utile pour la société

On a de bonnes vacances

On peut rencontrer des gens

On a des responsabilités

On fait un métier intéressant

On peut bien employer ses capacités

On peut voyager

On travaille en groupe

On a métier qui correspond à ses passions 
Pour terminer, voici quelques questions sociodémographiques qui nous permettront de mieux analyser vos réponses.

\section{Quel est votre sexe?}

Masculin

Féminin

\section{Quelle est votre année de naissance ?}

Je suis né(e) en $19 / \ldots$

\section{Avez-vous obtenu un baccalauréat...}

Général

Professionnel 2

Technologique 3

\section{Si vous avez obtenu un baccalauréat général, dans quelle section l'avez-vous obtenu ?}

Section ES 1

Section L 2

Section S option mathématiques 3

Section S option physique-chimie $\quad 4$

Section S option sciences de la vie et de la terre 5

$\begin{array}{lc}\text { Autre } & 6\end{array}$

56. Si vous avez obtenu un baccalauréat technologique, dans quelle section l'avez-vous obtenu

Sciences et technologies du management et de la gestion (STMG) 1

Sciences et technologies du design et des arts appliqués (STD2A) 2

Sciences et technologies de l'industrie et du développement durable (STI2D) 3

Sciences et technologies de laboratoire (STL) 4

Sciences et technologies de la santé et du social (ST2S) 5

Sciences et technologies du management et de la gestion (STMG) 6

Sciences et technologies du design et des arts appliqués (STD2A)

Sciences et technologies de l'agronomie et du vivant (STAV) 8

Technique de la musique et de la danse (TMD) 9

Technologie hôtellerie $\quad 10$

Autre 11 
57. En quelle année avez-vous obtenu votre baccalauréat ?

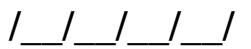

58. Depuis la seconde, dans quel type d'établissement avez-vous suivi vos études?

Lycée public uniquement

Lycée privé uniquement

Lycée public et lycée privé 3

Autre

59. Quelle est ou était (si la personne est en arrêt d'activité ou à la retraite) la profession de votre mère ? Précisez le plus possible.

60. Quelle est ou était (si la personne est en arrêt d'activité ou à la retraite) la profession de votre père? Précisez le plus possible.

61. Avez-vous des personnes proches qui travaillent dans le domaine scientifique ou technique ?

Oui

Non

Je ne sais pas

Nous vous remercions de votre participation.

Si vous souhaitez avoir des informations sur cette étude (questions, résultats...) vous pouvez contacter : Representations-chimie-biologie@univ-lille1.fr 


\subsection{La passation du questionnaire}

La diffusion du questionnaire s’est effectuée de début septembre à mi-octobre 2014 lors des réunions de prérentrée. Le questionnaire était proposé aux étudiants à l’issue de la réunion, sur un temps libre, pour une administration sur place. Conformément aux règles éthiques en vigueur, ils étaient libres de ne pas répondre et n’ont subi aucune pression les incitant à le faire.

Le taux de réponse élevé ${ }^{27}$ pourrait s'expliquer par le fait que les étudiants ont développé durant leur scolarité une « posture scolaire ${ }^{28}$ » qui les inciterait à répondre à des demandes faites par un membre de l'institution. Toutefois, cette hypothèse - bien qu'elle puisse avoir une incidence sur ce taux de retour - ne nous parait pas pleinement satisfaisante, car nous avions pris un très grand soin à présenter notre démarche comme non obligatoire, liée à un projet de recherche. Nous avions insisté à plusieurs reprises sur ce point. Ce taux de retour suggère surtout, selon nous, que les étudiants avaient de l'intérêt pour les thèmes proposés. Les réponses aux questions ouvertes ${ }^{29}$ - qui demandent de définir des concepts - renforcent cette interprétation.

\section{ANALYSE DES QUESTIONS FERMEES}

Les réponses aux questions fermées 5, 8, 9, $10^{30}$ ont fait l’objet d’analyses univariées et bivariées. Des tests de significativité, ainsi que des calculs de coefficients de corrélation ont systématiquement été réalisés. L’ensemble des données présentées est donc statistiquement valide. Par ailleurs un indice cumulatif permettant de classer en profils les individus en fonction de leur niveau d'intérêt pour le domaine scientifique a été constitué à partir des questions 8, 9 et 10. Il compte les enquêtés ayant répondu :

•très ou assez intéressé par les sujets scientifiques (q8) ;

•très souvent ou la plupart du temps informé par les sujets scientifiques (q9) ;

•plutôt enthousiastes avec les découvertes scientifiques (10).

On obtient ainsi un indice à quatre positions (de 0 à 3). Les individus qui n’ont répondu à aucune des modalités signalés ci-dessus sont groupés en position 0 (Intérêt - -) alors que ceux qui ont répondu à toutes les modalités sont classés dans le groupe 3 (Intérêt ++). En position 1 (Intérêt -) ou 2 (Intérêt +) figurent des individus avec des réponses intermédiaires.

\section{ANALYSE DES QUESTIONS OUVERTES}

Les réponses à la question 6 Pour vous, qu'est-ce que la science ? ont été saisies manuellement et ont fait l'objet d'une analyse statistique associant :

\footnotetext{
${ }^{27}$ Cf supra.

${ }^{28}$ « La posture scolaire caractérise davantage la manière dont l'élève essaie avant tout de rentrer dans les normes scolaires attendues, tente de se caler dans les attentes du maître. La posture ludique-créative traduit, elle, la tentation toujours latente et plus ou moins assurée de détourner la tâche ou de la re-prescrire à son gré. » Bucheton et Soulé 2009.

${ }^{29}$ Voir les questions 6, 20, 28, 35 et 44.

${ }^{30}$ Voir les libellés des questions dans le point 2.1 de ce document.
} 
- $\quad$ les fréquences des mots et expressions les plus couramment utilisés (en regroupant les mots de racine identique) ;

- $\quad$ une analyse de concordance.

Cette analyse a permis d'amorcer la construction d'une grille de thèmes pour faire une analyse de contenu. La grille a été enrichie par les thèmes identifiés par les travaux d'épistémologie et de philosophie des sciences ${ }^{31}$, ceux des études sur les rapports entre science et société (Public Awareness of Science, Public Understanding of Science, Public Engagement with Science and Technology) ${ }^{32}$ ainsi que ceux des travaux sur les objectifs éducatifs (Nature of Science et Features of Science) ${ }^{33}$. Cet apport a permis de préciser les différents thèmes liés à « ce à quoi sert la science » et « comment faiton de la science » quand l'analyse statistique complétait ces thèmes avec « ce que la science étudie ». Le contenu de réponses a ensuite été codifié ce qui a permis, par trois itérations successives, de définir la grille de thèmes finalement utilisée pour l'ensemble du corpus. À l'issue de ce codage, la grille a été associée aux données quantitatives afin de chercher les corrélations éventuelles.

\section{Bibliographie}

Bensaude-Vincent B. et al. (Eds.). (2017). « Research objects in their technological setting », Abingdon, Routledge.

Bucheton, D. et Soulé, F. (2009). Les gestes professionnels et le jeu des postures de l'enseignant dans la classe : un multi-agenda de préoccupations enchâssées. Éducation et didactique, 3(1), 29-48.

Matthews, MR. (2012) Changing the Focus: From Nature of Science (NOS) to Features of Science (FOS) In M.S. Khine (ed.), Advances in Nature of Science Research, (pp. 3-26). Dordrecht: Springer. Dagher, ZR. et Erduran, S. (2016). Reconceptualizing the Nature of Science for Science Education Why Does it Matter? Science et Education, 25, 147-164.

\footnotetext{
${ }^{31}$ La bibliographie est trop abondante et trop riche en textes fondamentaux pour pouvoir les citer de manière exhaustive. On peut cependant consulter Bensaude-Vincent 2017 dont nous nous sommes beaucoup inspirés, car cet ouvrage aborde conjointement la question de la nature de la science et des technosciences (forme récente prise par certaines technologies). ${ }^{32}$ Voir l'ensemble des rapports du Wellcome Trust (https://wellcome.ac.uk/) et du Parliamentary Office Science and Technology (https://www.parliament.uk/post). Voir par exemple le rapport de 2018 qui décrit les résultats et la méthodologie.

${ }^{33}$ Pour une synthèse, on peut citer Matthews 2012 et Dagher et Erduran 2016.
} 